\title{
An Exploratory Analysis of a Treatment Based Classification Algorithm to Treat Patellar Tendinopathy
}

\author{
Monica Matocha, DAT, LAT, ATC*; Patti Syvertson, DAT, ATC †; Janet McMurray, DAT, LAT, ATC $\ddagger$; Emily R. \\ Dietz, DAT, LAT, ATC, CEIS**, Russell T. Baker, PhD, DAT, AT, CMP, PRT-c®§; Alan Nasypany, EdD, LAT, \\ ATC§; Don Reordan, PT, MS, OCS, MCTA, CIDN€; Darcy Downey EdD, LAT, ATC $¥$ \\ *Texas Lutheran University, Seguin, TX; ${ }^{\top}$ Crystal Springs Upland Schools, Uplands, CA; $¥$ McMurry University, Abilene, TX; \\ **Elizabethtown College, Elizabethtown, PA; § University of Idaho, Moscow, ID; €Jacksonville Physical Therapy, Jacksonville, FL; \\ ¥Texas State Universtiy, San Marcos, TX
}

\begin{abstract}
The general and athletic populations commonly experience patellar tendon pain, which is frequently treated with a gold standard 12-week eccentric exercise protocol. The present research study was designed to determine the effects of a treatment based classification (TBC) algorithm utilizing indirect treatment techniques in patellar tendinopathy participants. Ten participants (seven females, three males, mean age $=19.6 \pm 1.07$, mean symptom duration $=2.14$ years with a range of one week to six years) with patellar tendinopathy were evaluated and included in this study. Each participant underwent a thorough evaluation process to aid in determining inclusion: participant medical history, range of motion measurements, orthopedic tests, a scan for soft tissue tender points, neurodynamic tests, and a local Mulligan Concept technique to determine diagnosis, study inclusion, and treatment classification. The following outcome measures were collected to establish baseline scores and assess participant improvement: the Disablement in the Physically Active Scale (DPA Scale), Numerical Rating Scale (NRS), Victorian Institute of Sports Assessment for the Patellar Tendon (VISA-P), Global Rating of Change (GRC), Nirschl Phase Rating Scale, and Blazina Knee Scale. Paired $t$-tests with $95 \%$ confidence intervals, were analyzed on NRS, DPA Scale, and VISA-P to determine the effectiveness of all treatment from initial exam to discharge. Cohen's $d$ was also computed to determine the effect size of each of the aforementioned outcome measures. Descriptive statistics were computed for the GRC at discharge. The mean change for the NRS ( $M=4.7,95 \% \mathrm{CI}[3.57$ to 5.82$], p<.001)$, DPA Scale $(M=21.8,95 \% \mathrm{CI}[12.43$ to 31.16$], p=.001)$, and VISA-P $(M=22.70,95 \% \mathrm{Cl}[33.71$ to 11.68$], p<.001)$ were statistically significant. The mean for the GRC (M = 5.3) was clinically meaningful. All of the participants $(100 \%)$ met discharge criteria. The results of this case series demonstrated an increase in function and decrease in pain for participants with patellar tendinopathy within three office visits when utilizing a TBC algorithm.
\end{abstract}

Key Phrases

Patellar tendinopathy, manual therapies, treatment-

based classification, patient-reported outcomes

\section{Correspondence}

Dr. Monica Matocha, Texas Lutheran

University, 1000 W Court St, Seguin, TX

78155

E-mail: mamatocha@tlu.edu.

Copyright (C) by Indiana State University All rights reserved. ISSN Online 2577-8188
Full Citation

Matocha M, Syvertson P, McMurray J, Dietz ER, Baker RT, Nasypany A, Reordan D, Downey D. An exploratory analysis of a treatment based classification algorithm to treat patellar tendinopathy. Clin Pract Athl Train. 2021;4(2): 6-20. https://doi.org/10.31622/2021/0004.2.2.

Submitted: April 3, 2019, 2020 Accepted: February 1, 2021.

\section{INTRODUCTION}

T endon related pathologies comprise 30 to $45 \%$ of sport related injuries, ${ }^{1}$ and frequently cause impairment in the general population. ${ }^{2}$ Patellar tendinopathy accounts for 7 to $40 \%$ of tendon related pathologies in sport ${ }^{3}$ and is characterized clinically by tendon pain, tendon dysfunction, 4,5 decreased performance in association with tendon swelling, morning stiffness, $4,6,7$ palpable crepitus, $3,6,7$ and localized swelling. ${ }^{4}$ Pain over a tendon is the key clinical diagnostic criteria used by clinicians to diagnose tendinopathy. 8 The use of advanced diagnostic imaging/testing (e.g., diagnostic ultrasound) is not common clinically, but is necessary to determine the exact physical state of the tendon. ${ }^{5}$

Though the clinical exam is the accepted standard for tendinopathy diagnosis, varying patient presentations and injury states make it difficult to identify the origin of tendon pain.9 Previously, tendon pain was thought to be a mechanical overuse injury, which caused inflammation in the tendon, and was classified as a tendinitis. ${ }^{10}$ Due to a lack of inflammatory markers being present

Clinical Practice in Athletic Training Volume 4 - Issue 2 - September 2021 
during histological tests, the term tendinopathy has generally become the preferred diagnostic term for tendon pain, 2,11 while tendinosis is utilized for a degenerative tendon diagnosed using diagnostic imaging. ${ }^{2}$ As tendon pathology research has elucidated other causative factors for the presentation of tendon pain (e.g., mechanical, neural, vascular), ${ }^{10}$ other researchers have proposed the use of the terms reactive tendinopathy and tendinalgia when classifying a patient with tendon pain. 12,13 The use of the term tendinalgia would allow clinicians to acknowledge the patient complaint of pain at the site of a tendon without predetermining a state of tissue pathology. ${ }^{13}$

The risk of using terminology focused on a specific causative factor is that it may lead to treatments that are not optimal for a specific patient or situation. 9 Due to the previous acceptance of an inflammatory condition being present when diagnosed as tendinitis, most interventions have been aimed at treating the inflammatory process. Most of these strategies do not produce effective long-term results (i.e., improvement past six weeks).14-16 Commonly used conservative treatments for patellar tendinalgia include: rest, nonsteroidal anti-inflammatory drugs (NSAIDs), stretching, eccentric exercises, and corticosteroid injections. $2,7,17$ The current treatment gold standard is the use of eccentric exercises. The Alfredson et al. protocol has become the foundation of most eccentric exercise protocols with participants performing the exercises two times a day, seven days a week, for 12 weeks. ${ }^{18-}$ 24 For many patients, however, compliance is difficult due to the length of the treatment, muscle soreness, and/or the pain experienced with treatment. 18,23,25 Other concerns with the protocol, such as tendon rupture rates, are not well understood as researchers do not always report treatment complications. Upon return to activity, participants who complete the protocol also report a high recurrence rate. 15,26

Another treatment option is to utilize manual therapies theorized to address the different

Copyright $(\subsetneq$ by Indiana State University All rights reserved. ISSN Online 2577-8188 causative factors of tendon pain; however, few research studies have been conducted to assess the effectiveness of manual therapy for the treatment of patellar tendinalgia. While there are a variety of manual therapy options that have been proposed to treat this disorder, clinicians could theoretically address the causative factors by applying the Mulligan Concept (MC), Positional Release Therapy (PRT), and/or neurodynamics in these cases. The MC techniques for knee dysfunction are based on applying a pain free glide (mobilization) to the joint while the patient actively moves into a position that was painful prior to the glide being applied. ${ }^{27}$ Positional Release Therapy (PRT) is theorized to restore the muscle or tendon to normal function by increasing oxygen and decreasing inflammatory metabolites. ${ }^{28}$ To determine if a peripheral neural sliding or tension dysfunction is present, neurodynamics is performed. Neurodynamics is the movement of the nervous system on other body structures. ${ }^{29}$ The use of these techniques in isolation, or combination, might better target the individual differences in patient presentation.

The use of manual therapies and tendon classification have been proposed as a means to improve the treatment of tendon pain ${ }^{12,13}$ due to the high rate of tendon pathology recurrence 15,26 and patient non-compliance. ${ }^{23,25}$ Researchers have proposed that many patients classified with tendinalgia may not actually have a true tissue pathology that must be addressed with tissue remodeling ${ }^{13}$ and that classifying patients based on their response to sub-therapeutic doses of intervention techniques may improve patient outcomes.9,13 Thus, it is important for clinicians to consider alternative examination and treatment strategies to better identify and treat these patients. The purpose of this study was to determine if a novel treatment based classification (TBC) algorithm could be used to classify tendon pain, participants, and what the effects of using the algorithm would be in participants diagnosed with patellar tendinalgia.

\section{PARTICIPANTS}




\section{Case Description}

A convenience sample of participants diagnosed by athletic trainers with patellar tendinalgia at four clinical sites across the United States of America participated in the study. The Texas Lutheran University Institutional Review Board approved the research project. All participants signed an informed consent form; if the participant was under the age of 18 years old, the legal guardian signed the informed consent and assent was provided by the minor. During the evaluation period, a total of 10 participants (seven females, three males, mean age $=19.6 \pm$ 1.07 , mean symptom duration $=2.14$ years with a range of one week to six years) presented for possible inclusion in the study. All of the potential participants were diagnosed with patellar tendinalgia according to the inclusion criteria (Table 1), agreed to participate in the study, and completed the study through discharge. All participants reported with patellar tendon pain, increased pain and stiffness in the morning and after sitting for long periods of time with a decrease in symptoms after warm up for physical activity. ${ }^{35}$

\section{Examination}

Each participant was examined using a predetermined clinical evaluation to ensure consistency in patellar tendinalgia diagnosis and classification with the TBC Algorithm. Inclusion criteria included: tendon pain before, during, or after patella loading activities; point tenderness over the patellar tendon upon palpation; pain near patella origin; impaired function; and tendon focal or generalized swelling. Exclusion criteria included: cortisone injection (<six weeks), fluoroquinolones ciprofloxacin use ( $<12$ months), post-operative participants unable to perform the treatment (<eight weeks), wore orthotics, currently healing or suspected fractures, or receiving physical therapy for the tendon of concern. Participants who met the inclusion criteria then completed a thorough history, range of motion (ROM), and special test examination. Special tests

Copyright $(\subsetneq$ by Indiana State University All rights reserved. ISSN Online 2577-8188 performed included: Clark's sign, patellar grind, patellar compression, prone knee bend, slump, a quarter screen for tender points (TP), 28 and the application of the $M C$ technique for the knee (an internal rotation glide followed by an external rotation glide if pain was not resolved during application). ${ }^{27}$ Clark's sign, patellar grind, and patellar compression tests were performed to rule out patellar dysfunction as the source of pain. The prone knee bend and slump tests were performed to rule in neurological tension and sliding dysfunctions. ${ }^{29}$ The quarter screen was performed to determine the presence of TPs; while the MC Technique was performed last to determine classification into the MC treatment.

\section{Treatment-Based Classification Algorithm}

The TBC algorithm consisted of a $M C$ technique, PRT, neurodynamics, and eccentric exercise. If the participant reported a resolution of his or her symptoms when the MC technique was applied during the exam, then the participant was classified as being a responder to the $M C$ treatment. If the application of the MC did not resolve symptoms during the exam and the participant presented with TPs in the lower extremity, which could be reduced by moving the participant into a position of comfort (POC), then the participant was classified as being a responder to the PRT treatment. If the application of the MC did not resolve symptoms and a POC could not be identified with PRT, the participant would be classified into the neurodynamic treatment if a positive neurodynamic test was found during the initial exam. In the case where the participant could not be classified into the MC, $P R T$, or neurodynamic group, the participant was classified into the eccentric exercise treatment protocol (Figure 1).

Once the clinician determined the appropriate treatment classification, the participant underwent three bouts of treatment within 10 days. The participant was re-assessed to determine if discharge criteria had been met at the conclusion of the third visit. Discharge criteria included: phase 
Table 1. Participant Presentation and Treatment

Participant \#

\section{Symptoms}

Pain mid-patellar tendon; unable to sit for more than 30 minutes without pain; pain interferes with competition; intermittent pain at rest; increase pain with stairs and squats; worst pain in the morning

Participant 2 Pain mid-patellar tendon; pain interferes with competition; intermittent pain at rest; increase pain with stairs, cutting, and squats; worst pain in the morning

Participant 3 Pain slightly inferior to patella; increase pain when sitting with knees bent, squatting, lunging, going up stairs; pain at start of activity; worst pain in the morning

Participant 4 Pain slightly inferior to patella; increased pain when sitting with knees bent longer than 30 minutes, squatting, lunging, and going upstairs; pain at start of activity; worst pain in the morning

Participant 5 Pain mid-patellar tendon; pain during ADLs, lunging, stairs, single leg hops; pain at onset of activity but able to perform; difficulty maintaining same position for extended period of time; pain at start of activity; worst pain in the morning

Participant 6 Pain mid-patellar tendon; pain during ADLs, lunging, stairs, single leg hops; pain at onset of activity but able to perform; difficulty maintaining same position for extended period of time; worst pain in the morning

Participant 7

Pain inferior to patella; unable to sit longer than 10 minutes without pain; unable to perform squats due to pain; difficulty with running and jumping; pain with ADLs and competition; worst pain in the morning

Participant 8

Pain slightly superior to tibial tuberosity; unable to sit longer than 10 minutes without pain; unable to perform squats due to pain; difficulty with running and jumping; pain with ADLs and competition; worst pain in the morning

Participant 9 Pain mid-patellar tendon; unable to perform single leg hops due to pain; difficulty with running and jumping; unable to compete due to pain; worst pain in the morning

Participant 10 Pain slightly superior to tibial tuberosity; intermittent pain at rest; unable to compete due to pain; worst pain in the morning

ND: neurodynamics, TP: tender point, Positive MWM: achieved PILL effect; ADLs: activities of daily living
Symptom Duration

Positive Special Tests

1 Week

2 Weeks

2 Months

2 Months

6 Months

6 Months

4 Years

4 Years

6 Years

TP: patella, patellar tendon, $A C L$, and medial hamstring

TP: patella and patellar tendon MWM: lateral rotation - squat
Copyright $(\odot$ by Indiana State University

All rights reserved. ISSN Online 2577-8188
9

Clinical Practice in Athletic Training

Volume 4 - Issue 2 - September 2021 
1 on the Nirschl Phase Rating Scale, phase 1 on the Blazina Knee Scale, and met minimal clinical important difference (MCID) for Global Rating of Change (GRC), and acute MCID for the Disablement of the Physically Active Scale (DPA Scale). Additionally, participants had to report a worst pain score equal to or less than two out of ten on the Numerical Rating Scale (NRS) during the discharge evaluation. If the participant was not discharged, a re-evaluation using the TBC algorithm was conducted to determine the participant's treatment classification for the next three visits. The participant was only able to be re-classified into the initial treatment classification if the participant demonstrated enough improvement to meet $50 \%$ of each discharge outcome criteria; if not, the participant was classified into the eccentric exercise treatment. Following discharge, each participant was sent a one-month follow up survey to collect follow-up scores on the NRS post-discharge.

\section{OUTCOME MEASURES}

Disablement and global participant outcome measures were utilized in this study to determine participant perceptions of their condition and recovery. The six outcome measures utilized in this study were the: NRS, GRC, DPA Scale, VISA-P, Nirschl Phase Rating Scale, and the Blazina Knee Scale.

The NRS is a rating scale a clinician can utilize to determine a participant's perception of his or her pain from zero, no pain, to ten, worst pain imaginable. ${ }^{30}$ Each participant of this case series was asked to rate his or her pain at best, worst, and rest before and after each treatment. The recorded NRS scores represent the participant's reported worst pain. The participant was also asked to rate his or her pain while the clinician performed a quarter screen for TPs. The GRC was utilized to determine participant's perception of his or her improvement or deterioration over time. ${ }^{31}$ The GRC was reported at every third visit for each participant. The (MCID) has been

Copyright (C) by Indiana State University All rights reserved. ISSN Online 2577-8188 established at two points for both the NRS 32 and GRC. ${ }^{31}$

The DPA Scale was developed to determine the participant's perception of how his or her injury has effected disablement. ${ }^{33}$ A participant reported his or her perception on a scale of one, no problem, to five, severe, on 16 questions across multiple domains: pain, motion, muscular function, stability, changing directions, daily actions, maintaining positions, skill performance, overall fitness, participation in activities, and well-being. The rating for each item on the scale is summed and 16 points are subtracted to produce a final score that ranges from zero to 64 points. The DPA Scale was administered upon the first visit, third visit, and every third visit after until discharged. The MCID has been established for the DPA Scale as nine points for acute injuries and six points for chronic injuries. ${ }^{33}$ The range of scores for healthy patients on the DPA Scale has been reported to be between zero and 34 points. ${ }^{33}$

The VISA-P was created to determine functional impairment in a participant with patellar tendon pain. ${ }^{34}$ The participant recorded responses to questions regarding his or her function on a numerical scale from zero, unable to perform, to ten, fully functional. All responses were then summed and recorded on a scale from zero, no function, to 100, fully functional. Each participant recorded VISA-P score upon the first visit, third visit, and discharge visit. Currently, a MCID has not been established for VISA-P.

The Nirschl Phase Rating Scale and Blazina Knee Scale were both developed to help classify participant symptoms. The Nirschl Phase Rating Scale was created for all tendon pain participants, 2,35 whereas the Blazina Knee Scale was created to determine dysfunction specifically for participants with patellar tendon pain. ${ }^{36}$ All participants reported his or her symptoms in accordance with both scales upon the first visit, third visit, and discharge visit. Currently, the Nirschl Phase Rating Scale, and Blazina Knee 
Figure 1. Treatment Based Classification Algorithm for Patellar Tendinopathy

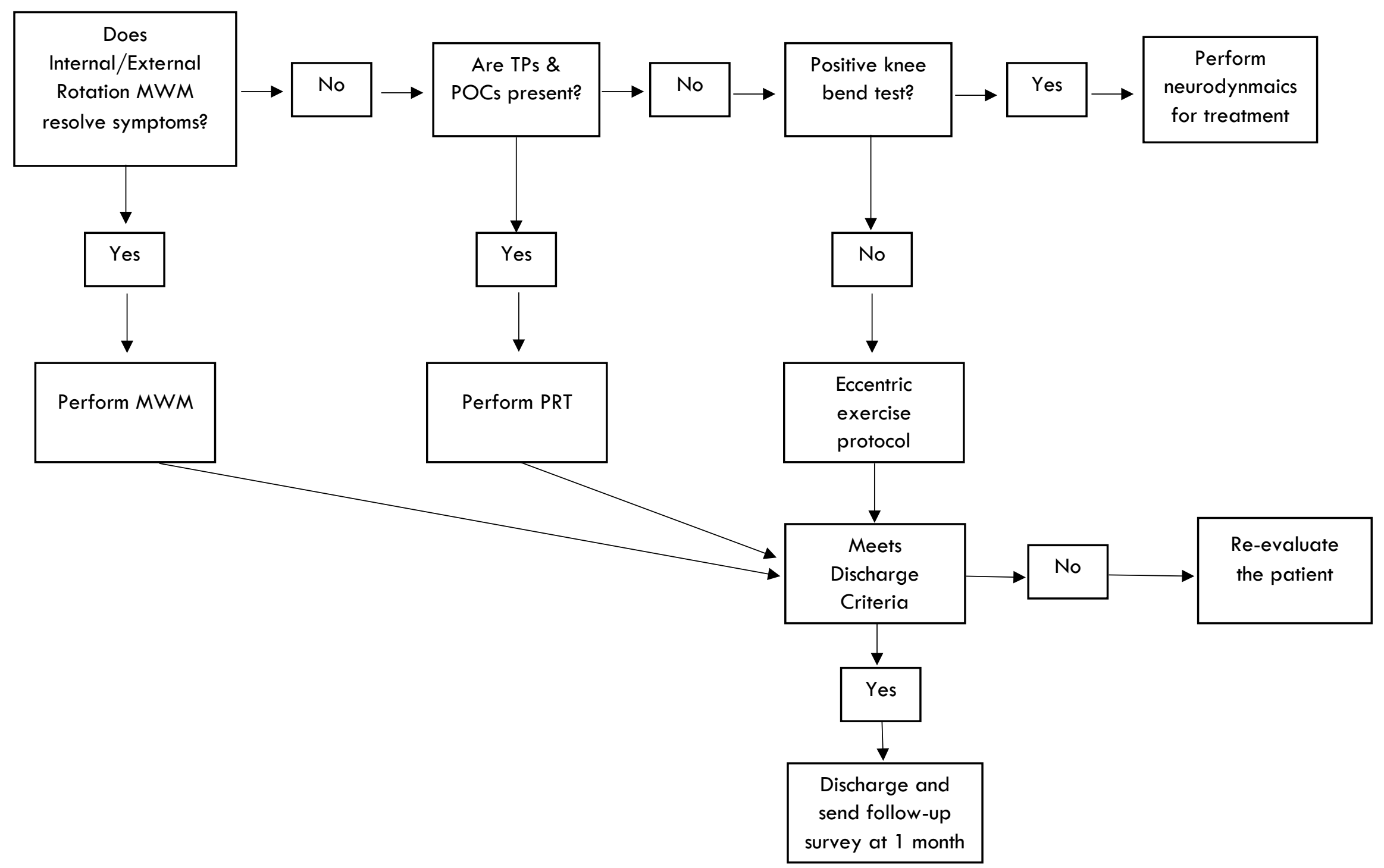


Scale do not have an established method for evaluating patient improvement on the scales.

\section{INTERVENTION}

If the participant was classified into the $M C$ treatment subgroup, the participant was treated with the Mobilization with Movement (MWM) (internal or external rotation glide) to resolve the participant's pain complaint. ${ }^{27}$ The internal or external rotation glide was applied by having the participant perform a movement that exacerbated the chief complaint. Once the painful movement was established, the clinician gently placed her hands just below the tibiofemoral joint line, around the tibia and fibula and applied the appropriate glide to the tibia in association to the femur (Figure 2). Simultaneously, the participant performed the previously established painful movement. The MWM was performed through three sets of 10 repetitions of pain-free movement. ${ }^{27}$

If the participant was classified into the PRT treatment subgroup, the dominant TP was monitored while the participant was passively moved into a POC. ${ }^{28}$ The clinician would begin with the participant in a supine position on a plinth with a bolster under their ankle to allow full knee extension. The clinician would then apply tibial rotation with the hand not palpating the TP. The POC was defined as a position resulting in the resolution of pain (zero out of 10 on the NRS) during palpation of the TP. If a POC was achieved, the participant received PRT for the dominant TP only. The dominant TP was treated while the clinician maintained the POC (Figure 3). The POC was held for a minimum of 30 seconds, and a maximum of 90 seconds. ${ }^{28}$ The participant was then returned to the normal anatomical position while the clinician continued monitoring the TP. The TP was reassessed by determining pain to palpation (using the NRS) in the normal anatomical position. If the participant still

Copyright (C) by Indiana State University All rights reserved. ISSN Online 2577-8188 reported tenderness to palpation of the TP after one set of treatment, the clinician repeated the treatment; if the patient reported resolution of pain to palpation, the treatment was concluded for that session. A patient could receive a maximum of three treatment sets per visit.

If the participant was classified into the neurodynamic treatment subgroup, the participant was instructed on the proper technique to perform a general neural slider in the prone knee bend position (Figure 4).29 As the participant released tension at the head (head moved from cervical extension to neutral), tension was increased at the knee (knee moved from extension to flexion). ${ }^{29}$ Each participant completed three sets of 10 repetitions, through a slow and controlled movement.

If the participant was classified into Eccentric Exercise (EE) treatment subgroup, the participant completed a monitored EE protocol two times a day, seven days a week for 12 weeks. ${ }^{37}$ Participants completed one set of 15 repetitions of a single leg squat on a 25-degree decline board for each session. ${ }^{37}$ The participant was instructed to keep the trunk in the upright position, slowly flexing the knee to 90 degrees and returning to the starting position with the uninjured leg. ${ }^{37}$ The participant was then instructed to squat into pain without exceeding seven out of ten on the NRS during the eccentric portion (knee flexion). If the participant's pain decreased to less than or equal to two out of ten on the NRS while performing $\mathrm{EE}$, an external load by use of a dumbbell was added to increase the difficulty of the exercise. 


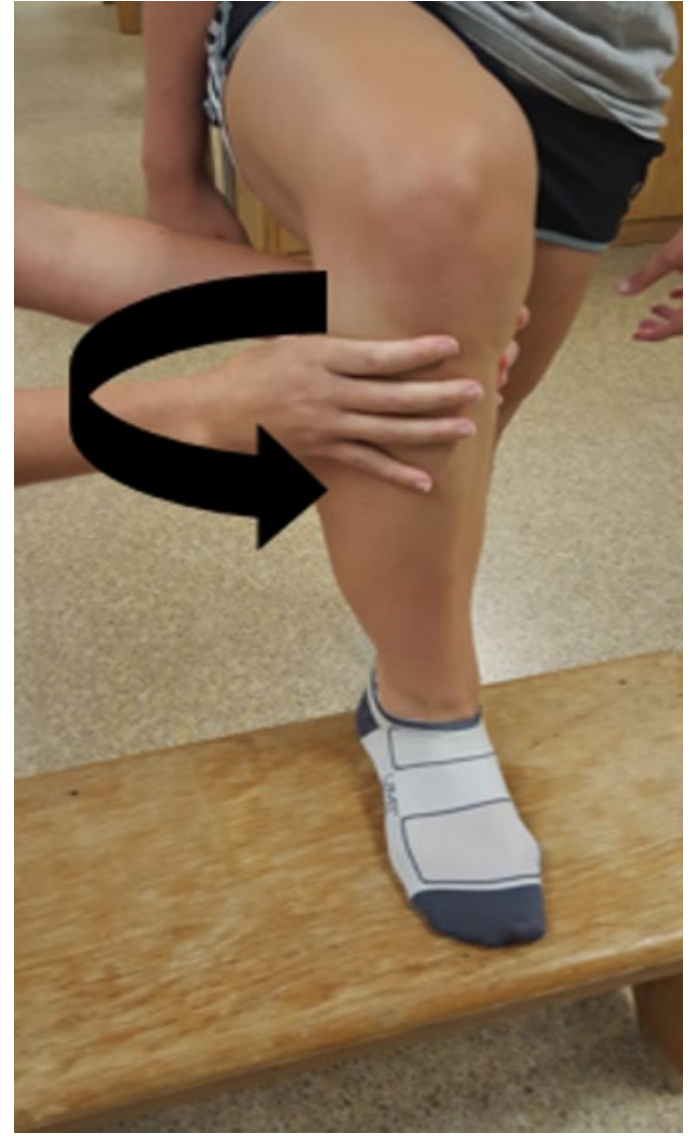

Figure 2. Example of the Mobilization with Movement

\section{Statistical Analysis}

All data was analyzed using SPSS version 23.0 (SPSS Inc., Chicago, IL, USA). Paired t-tests were performed on the NRS, DPA Scale, and VISA-P to determine the effects of classifying and treating participants with this novel TBC algorithm for patellar tendinalgia. Mean differences from the initial visit scores and $95 \%$ confidence intervals (Cls) were calculated for the NRS, DPA Scale, and VISA-P for discharge. Cohen's $d$ was also computed to determine the effect size, or maximum likelihood, of each of the aforementioned outcome measures. For Cohen's d an effect size of 0.2 to 0.3 was considered a "small" effect, around 0.5 a "medium" effect and 0.8 to infinity, a "large" effect. ${ }^{38}$ Descriptive statistics were performed on the GRC scores reported at discharge.

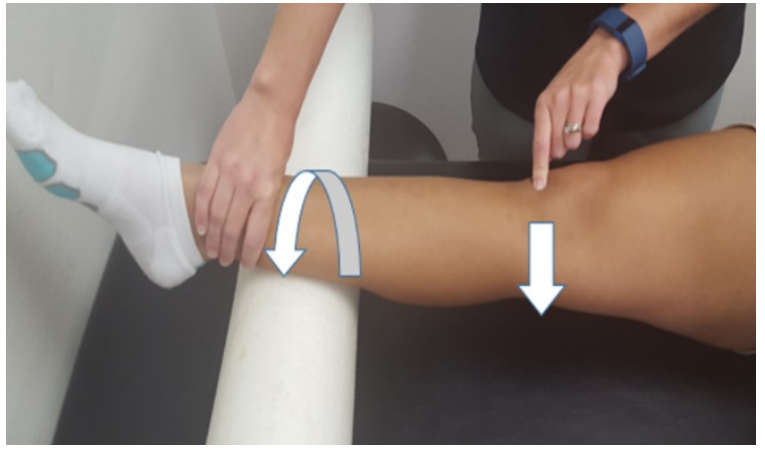

Figure 3. Example of Positional Release Therapy Position of Comfort

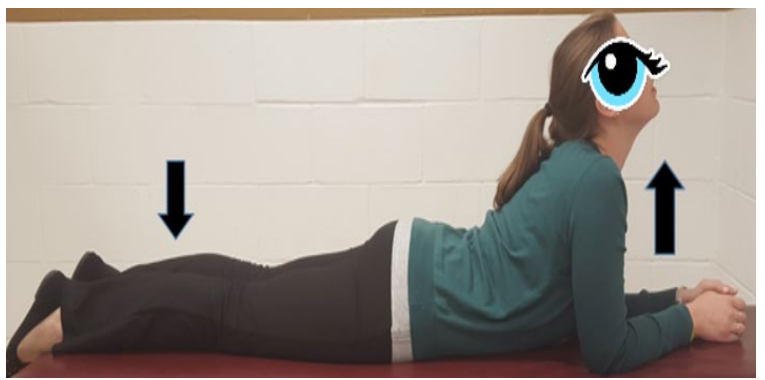

Figure 4. Example of Neurodynamic Slider Technique

\section{RESULTS}

During the initial examination, all participants were classified into a manual therapy treatment sub-group $(M C=9, P R T=1)$. All participants were successfully treated through discharge with the initial treatment classification and no participants met the criteria for classification into the EE subgroup at any point of time during treatment. The number of treatments each participant received was three over a mean of $4.8 \pm 1.4$ days to discharge.

\section{Numerical Rating Scale}

The use of the TBC algorithm resulted in a significant mean change in pain from initial visit to discharge, $M=4.7 \pm 1.64(95 \% \mathrm{Cl}[3.57$ to 5.82], $p<.001$ ) with a large effect size (Cohen's $d=2.41$ ) (Table 2). The mean difference in pain Volume 4 - Issue 2 - September 2021 
scores from initial visit to discharge, as well as the lower boundary $\mathrm{Cl}$, exceeded the MCID of "much better" for the NRS. ${ }^{26}$ The mean change was accomplished in just three visits that took place within $4.8 \pm 1.4$ days. At discharge, $60 \%$ of participants $(6 / 10)$ reported a complete resolution of their pain. The remaining $40 \%$ of participants $(4 / 10)$ reported their "worst" pain as a one $(20 \%, 2 / 10)$ or two $(20 \%, 2 / 10)$ on the NRS. One-month post discharge data demonstrated that all participants who completed the follow-up survey ( $\mathrm{n}=2$ ) continued to experience a resolution of pain with full return to activity.

\section{Disablement in the Physically Active Scale}

Statistically significant changes on the DPA Scale from initial evaluation to discharge were recorded $M=21.8 \pm 12.3130,(95 \% \mathrm{Cl}$ [12.43 to 31.16], $p=.001$ ), with a large effect size (Cohen's $d=$ 1.98) when using the TBC algorithm (Table 2). The mean change from initial visit to discharge, as well as the lower boundary of the $\mathrm{Cl}$, exceeded the MCID for acute conditions, a reduction of nine points or greater, which is greater than the MCID for chronic conditions (six points). ${ }^{33}$ All of the participants (100\%) met MCID for both acute and chronic conditions prior to discharge, as well as being discharged within the healthy range (zero to 34 points). 33 Published data for DPA Scale scores for return to activity for chronic conditions does not exist; however, the reported mean for participants who returned from acute injury is $M=$ $8.82 \pm 6.71$ ( $R=0-23$ points). All of the participants $(100 \%)$ in this case series were discharged below the reported mean score for returning to activity after an acute injury $(M=8.5$ $\pm 9.11 ; R=0-22$ ). Consequently, participants in this case series perceived less disablement than has been reported in the previous literature on the DPA Scale. ${ }^{33}$

\section{Victorian Institute Sport Assessment - Patella}

The use of the TBC algorithm resulted in a significant increase in scores on the Victorian

Copyright $\left({ }^{\circ}\right.$ by Indiana State University All rights reserved. ISSN Online 2577-8188
Institute of Sport Assessment-Patella outcome measure from initial exam to discharge $(M=$ $22.70 \pm 16.07,95 \% \mathrm{Cl}[33.71$ to 11.68$], p<$ .001 ), with a large effect size (Cohen's $d=1.37$ ) (Table 2). Of greater clinical importance, $80 \%$ $(8 / 10)$ of the participants reported a VISA-P score for "completely recovered" within three days of initiating treatment.

\section{Global Rating of Change}

A clinically meaningful increase on GRC scores from initial visit to discharge was reported ( $M=$ $5.7 \pm 2.11$ ) (Table 2). The GRC scale ranges from -7 (a very great deal worse) to +7 (a very great deal better). ${ }^{31}$ All $(100 \%)$ of the participants exceeded a MCID for the GRC scale $(\geq 2)$ upon discharge. ${ }^{31}$ More clinically relevant, $50 \%(5 / 10)$ of participants reported $a+7$ (a very great deal better), $10 \%(1 / 10)$ reported a +6 (a great deal better), and $40 \%(4 / 10)$ reported a +4 (moderately better) at discharge. ${ }^{31}$

\section{Nirschl Phase Rating Scale}

During initial evaluations, $30 \% \quad(3 / 10)$ of participants reported a phase three on the Nirschl Phase Rating Scale meaning "pain that is present during activity without causing activity modification", ${ }^{35} 40 \%(4 / 10)$ reported a phase five "pain that is present during all activities and occurs with activities of daily living", 35 and $30 \%$ $(3 / 10)$ reported a phase six "intermittent rest pain that does not disturb sleep". 35 All participants $(100 \%, 10 / 10)$ reported a phase one ("mild stiffness or soreness after activity with resolution of symptoms within 24 hours") on the Nirschl Phase Rating Scale prior to discharge. More clinically relevant, $60 \%(6 / 10)$ of the participants did not feel a phase one rating on the Nirschl Phase Rating Scale was applicable due to their experience of full resolution of symptoms. 
Table 2. Outcome Results

\begin{tabular}{lcccccc}
\hline $\begin{array}{l}\text { Outcome } \\
\text { Measure }\end{array}$ & Intake Score & $\begin{array}{c}\text { Discharge } \\
\text { Score }\end{array}$ & Mean Change & $95 \%$ Cls & P-value & $\begin{array}{c}\text { Effect Size } \\
\text { (Cohen's d) }\end{array}$ \\
\hline NPRS & $5.30 \pm 1.94$ & $0.60 \pm 0.84$ & $4.78 \pm 1.64$ & $3.52,6.04$ & 0.000 & 2.41 \\
DPA Scale & $30.3 \pm 11.02$ & $8.50 \pm 9.12$ & $19.89 \pm 12.31$ & $10.42,29.35$ & 0.001 & 1.98 \\
VISA-P & $53.60 \pm 16.58$ & $76.30 \pm 18.36$ & $-23.56 \pm 16.71$ & $-35.91,11.20$ & 0.002 & 1.37 \\
\hline
\end{tabular}

NPRS: Numeric Pain Rating Scale; DPA Scale: Disablement of the Physically Active Scale; VISA-P:

Victorian Institute of Sport Assessment for Patellar Tendon; Cls: Confidence Intervals

\section{Blazina Knee Scale}

During the initial evaluation, $50 \%(5 / 10)$ of participants reported a phase two on the Blazina Knee Scale "pain/discomfort during and after activity with the subject still able to perform at a satisfactory level (does not interfere with participation)"; ${ }^{36}$ while the other $50 \%(5 / 10)$ of participants reported a phase three "pain during and after activity with more prolonged, with subject having progressively increasing difficulty in performing at a satisfactory level (interferes with competition)".36 All of the participants $(100 \%)$ reported a phase one on the Blazina Knee Scale prior to discharge ("pain after activity only"). ${ }^{36}$ More clinically relevant, $60 \%(6 / 10)$ of participants did not feel a phase one rating on the Blazina Knee Scale was applicable due to their experience of full resolution of symptoms.

\section{DISCUSSION}

Currently, eccentric exercise is the gold standard treatment for patellar tendinalgia. Several researchers have demonstrated positive results with the use of a 12-week protocol.18-21,23-25,38,39 Jonsson and Alfredson 40 compared an eccentric exercise group to a concentric exercise group for the treatment of "jumper's knee" and reported nine out of 10 participants who completed the study were "satisfied" and discharged with a mean Visual Analogue Scale (VAS) of 23 out of 100 and a VISA-P score of 83 points with the use of a 12-week eccentric exercise protocol. Similarly, Purdam, et al. ${ }^{24}$ reported a mean VAS score of 28.5 points at discharge for participants who performed eccentric exercises on a decline

Copyright (C) by Indiana State University All rights reserved. ISSN Online 2577-8188 board, compared to a mean VAS score of 72 points at discharge for participants who performed traditional squat eccentric exercises for 12 weeks. In these studies, however, not all participants reported being "satisfied" at discharge $\left(10 \%, 4025 \%{ }^{24}\right)$. The participants, who did report being "satisfied" did not, on average, experience a full resolution of pain at discharge after 12 weeks of therapy. ${ }^{24,40}$ Although NRS and VAS are measured on different scales, the values of the numbers are similar. The clinicians of the present study were able to discharge participants with mean NRS scores of .05 out of $10(0 / 10=6$, $1 / 10=2,2 / 10=2)$, which is lower than the aforementioned studies. Although the mean VAS of the present study was lower, the mean VISA-P $(M=78.11)$ of this study is also lower than the aforementioned study, which is potentially indicative of participant perception of more function from the EE protocol.

Although EE has been found to produce beneficial results when the protocol is completed, there are still concerns over the effectiveness of the protocols for all patients and a lack of a clear understanding of the mechanism of action. Thus, there is a need to determine if tendinalgia participants should be screened prior to using an EE protocol in a one-size fits all model.8,9 The lack of a screening process for identifying patients likely to respond to $E E$ and the extended time required for patients to become symptom free has created a need for improved assessment methods. 8,9 One potential solution to improve tendinalgia outcomes is the use of a TBC system or more novel manual therapy techniques. Lewis' has suggested a series of four mechanical techniques, Volume 4 - Issue 2 - September 2021 
or a combination of interventions, to be used as a TBC system to produce improved patient classification and treatment outcomes for patients with rotator cuff tendinalgia. The manual therapies used in the TBC algorithm in this study also have evidence of effectiveness on tendinalgia patients in other research studies. ${ }^{13,42-}$ 44

Researchers have found promising results with the use of the MC when treating lateral epicondylalgia. ${ }^{41-43}$ Bisset, et al. ${ }^{43}$ observed favorable outcomes for the use of MC mobilizations in combination with exercises over corticosteroids and a "wait and see" method. Although corticosteroid injections were more statistically significant

While the preliminary results of this case series are important, the limitations of this study must also be noted. Although many attempts were made to decrease the risk of bias, there could have been a bias created because the clinician and participants were not blinded to the treatment or collection of outcome measures. Additionally, a control or placebo group was not used in the study. The lack of control group and long term follow-up made it difficult to definitively determine if the outcomes were the result of treatment or the natural course of healing; however, a number of participants presented with chronic symptoms (mean symptom duration $=2.14$ years with a range of one week to six years) unlikely to have spontaneously healed over the treatment period. The lack of comparison group made it difficult to determine if one treatment intervention was superior to another within the TBC algorithm, but the purpose of the study was not to identify the "best" intervention. Instead, the focus was on determining the effectiveness of classifying patients using sub-therapeutic doses on indirect manual therapies. Additionally, it could be argued the treatments provided as part of the TBC algorithm were provided at sub-therapeutic doses (e.g., not treating multiple TPs with PRT, etc.) and the interventions could be more effective if treatment dose was maximized. Furthermore, the

Copyright $(\subsetneq$ by Indiana State University All rights reserved. ISSN Online 2577-8188 specific techniques utilized (e.g., internal rotation and external rotation $M W M$, etc.) for this $T B C$ algorithm were limited to increase usability of the algorithm; however, other techniques within the different intervention paradigms have the potential to maximize the effectiveness of each paradigm (e.g., MC taping technique, other glides, etc.). The full examination of the original TBC was not fully assessed due to the lack of diverse treatment subgroups. There were nine participants classified in the MC subgroup, one in the PRT subgroup, and zero in the ND and EE subgroups. The final limitation is that the participants may not have fully represented patellar tendinalgia patients and those who volunteered may have been motivated to improve.

As this study is an initial examination of a TBC algorithm for patellar tendon pain, it is possible that altering the order or adding other treatment paradigms may be appropriate to maximize the effectiveness of the TBC algorithm. The results of this study do provide support for the utilization of a TBC algorithm for patellar tendinalgia patients because all 10 participants experienced statistically and clinically significant improvements in pain and function in three visits. Future research should compare this TBC algorithm with a control or placebo group and utilize long-term follow-up with the participants. Forthcoming research should also include diagnostic imaging or histological exams, which would benefit the understanding of the physiological changes in the tendon following treatment utilizing the TBC algorithm.

\section{CLINICAL APPLICATION}

The TBC algorithm used in this study was designed because the clinicians could observe participant response to potential interventions while in a painful state and to utilize manual therapy techniques that could potentially produce rapid changes. Patient response enabled the clinician to classify the participant to an intervention that was designed to be matched to their dysfunction. In theory, matching tendinalgia patients to therapies 
through classification could improve outcomes. In this study, all of the participants were classified as being a responder to either the MC or PRT and were able to meet the pre-established discharge criteria without a single participant needing to be classified into the EE protocol sub-group at any time. Thus, a TBC algorithm may be more effective at matching participants to appropriate treatments that do not require extended therapy or a painful experience to produce effective outcomes. Additionally, the use of a TBC algorithm may allow clinicians to identify which participants actually need to participate in an EE protocol or when to add this protocol as an adjunct therapy to provide complete resolution of participant complaints.

\section{REFERENCES}

1. Murtaugh, B., Ihm J. Eccentric training for the treatment of tendinopathies. Curr Sports Med Rep. 2013;12(3):175-182. https://doi.org/10.1249/isr.0b013e31829 33761.

2. Kaux J-F, Forthomme B, Goff C Le, Crielaard J-M, Croisier J-L. Current opinions on tendinopathy. J Sports Sci Med. $2011 ; 10(2): 238-253$.

3. Fredberg U, Stengaard-Pedersen K. Chronic tendinopathy tissue pathology, pain mechanisms, and etiology with a special focus on inflammation. Scand J Med Sci Sports. $2008 ; 18(1): 3-15$.

https://doi.org/10.1111/i.16000838.2007.00746.x.

4. Khan KM, Cook JL, Bonar F, Harcourt $P$, Astrom M. Histopathology of common tendinopathies. Update and implications for clinical management. Sports Med. 1999;27(6):393-408.

https://doi.org/10.2165/00007256199927060-00004.

5. Rio E, Moseley L, Purdam C, et al. The pain of tendinopathy: Physiological or pathophysiological? Sport Med. 2013;44(1):9-23. https://doi.org/10.1007/s40279-0130096-z.

6. Alfredson H, Lorentzon R. Chronic tendon pain: No signs of chemical inflammation but high concentrations of the neurotransmitter glutamate. Implications for treatment? Curr Drug Targets. 2002;3(1):43-54. https://doi.org/10.2174/1389450023348 $\underline{028 .}$.

7. Alfredson H. The chronic painful Achilles and patellar tendon: research on basic biology and treatment. Scand J Med Sci Sports. $2005 ; 15(4): 252-9$. https://doi.org/10.1111/i.16000838.2005.00466.x.

8. Scott A, Docking S, Vicenzino B, et al. Sports and exercise-related tendinopathies: $A$ review of selected topical issues by participants of the second International Scientific Tendinopathy Symposium (ISTS) Vancouver 2012. Br J Sports Med. 2013;47(9):536-44.

https://doi.org/10.1136/bjsports-2013092329.

9. Lewis J. Rotator cuff tendinopathy/subacromial impingement syndrome: is it time for a new method of assessment? $\mathrm{Br} J$ Sport Med. 2008. https://doi.org/10.1136/bjsm.2008.05218 3 .

10. Rees JD, Wilson a M, Wolman RL. Current concepts in the management of tendon disorders. Rheumatology (Oxford). 2006;45(5):508-21. https://doi.org/10.1093/rheumatology/kel $\underline{046}$.

11. Mayor RB. Treatment of athletic tendonopathy. Conn Med. 2012;76(8):4715.

12. Cook JL, Purdam CR. Is tendon pathology a continuum? A pathology model to explain the clinical presentation of load-induced tendinopathy. $\mathrm{Br} J$ Sports Med. 2008;43:409-416. https://doi.org/10.1136/bjsm.2008.05119 3. 
13. Baker RT, Riper MV, Nasypany AM, Seegmiller JG. Evaluation and Treatment of Apparent Reactive Tendinopathy of the Biceps Brachii. Int $J$ Athl Ther Train. 2014;19(July):14-21.

https://doi.org/10.1123/ijatt.2014-0023.

14. Hay $E M$, Paterson $S M$, Lewis $M$, et al. Pragmatic Randomised Controlled Trial of Local Corticosteroid Injection and Naproxen for Treatment of Lateral Epicondylitis of Elbow in Primary Care. BJM; 1999;319(7215):964-968. https://doi.org/10.1136/bmi.319.7215.96 4 .

15. Smidt N, van der Windt $D$ a WM, Assendelft WJJ, Devillé WLJM, Korthals-de Bos IBC, Bouter LM. Corticosteroid injections, physiotherapy, or a wait-and-see policy for lateral epicondylitis: a randomised controlled trial. Lancet. 2002;359(9307):657-62. https://doi.org/10.1016/S01406736(02)07811-X.

16. Andres BM, Murrell $G$ a C. Treatment of tendinopathy: what works, what does not, and what is on the horizon. Clin Orthop Relat Res. 2008;466(7):1539-54.

https://doi.org/10.1007/s1 1999-0080260-1.

17. Glaser T, Poddar S, Tweed B, Webb CW. Clinical inquiries. What's the best way to treat Achilles tendinopathy? J Fam Pract. 2008;57(4):261-263.

18. Alfredson $H$, Pietilä $T$, Jonsson $P$, Lorentzon R. Heavy-load eccentric calf muscle training for the treatment of chronic Achilles tendinosis. Am J Sports Med. 1998;26(3):360-6. https://doi.org/10.1177/0363546598026 0030301.

19. Mafi N, Lorentzon R, Alfredson H. Superior short-term results with eccentric calf muscle training compared to concentric training in a randomized prospective multicenter study on patients with chronic Achilles tendinosis. Knee Surgery, Sport Traumatol Arthrosc. $2001 ; 9(1): 42-47$. https://doi.org/10.1007/s001670000148.

Copyright $(\subset)$ by Indiana State University All rights reserved. ISSN Online 2577-8188
20. Fahlström $M$, Jonsson $P$, Lorentzon $R$, Alfredson $\mathrm{H}$. Chronic Achilles tendon pain treated with eccentric calf-muscle training. Knee Surg Sports Traumatol Arthrosc. 2003;11(5):32733. https://doi.org/10.1007/s00167-0030418-z.

21. Jonsson $P$, Alfredson $H$, Sunding $K$, Fahlström $M$, Cook J. New regimen for eccentric calfmuscle training in patients with chronic insertional Achilles tendinopathy: results of a pilot study. Br J Sports Med. 2008;42(9):7469.

https://doi.org/10.1136/bjsm.2007.03954 $\underline{5}$.

22. Morrissey D, Roskilly A, Twycross-Lewis R, et al. The effect of eccentric and concentric calf muscle training on Achilles tendon stiffness. Clin Rehabil. 2011;25(3):238-47. https://doi.org/10.1177/0269215510382 600.

23. Dimitrios $S$, Pantelis $M$, Kalliopi S. Comparing the effects of eccentric training with eccentric training and static stretching exercises in the treatment of patellar tendinopathy. A controlled clinical trial. Clin Rehabil. $2011 ; 26(5): 423-30$.

https://doi.org/10.1177/0269215511411 114.

24. Purdam CR, Jonsson $P$, Alfredson $H$, Lorentzon $R$, Cook JL, Khan KM. A pilot study of the eccentric decline squat in the management of painful chronic patellar tendinopathy. $\mathrm{Br} J$ Sports Med. 2004;38(4):395-7. https://doi.org/10.1136/bjsm.2003.00005 3.

25. Svernlöv B, Hultgren E, Adolfsson L. Medial epicondylalgia (golfer's elbow) treated by eccentric exercise. Shoulder Elb. 2012;4(1):50-55. https://doi.org/10.1111/i.17585740.2011.00152.x.

26. Bisset L, Beller E, Jull G, Brooks P, Darnell R, Vicenzino B. Mobilisation with movement and exercise, corticosteroid injection, or wait and see for tennis elbow: randomised trial. $\mathrm{Br}$ Med J. 2006;333(7575):939-941. 
https://doi.org/10.1136/bmi.38961.58465

3.ae.

27. Mulligan BR. Manual Therapy NAGS, SNGAS, MWM etc. 6th ed. Wellington, New Zealand: Plane View Services Ltd; 2010.

28. D'Ambrogio K, Roth G. Positional Release Therapy, Assesment \& Treatment of Musculoskeletal Dysfunction. St. Louis: Mosby; 1997.

29. Shacklock M. Clinical Neurodynamics. London: Elsevier Inc.; 2005.

30. McCaffery M, Beebe A. Pain: Clinical Manual for Nursing Practice. St. Louis, MO: C. V. Mosby; 1989.

31. Kamper S, Maher C, MacKay G. Global Rating of Change scales. Aust J Physiother. 2009;55(4):289.

https://doi.org/10.1016/500049514(09)70015-7.

32. Farrar JT, Young JPJ, LaMoreaux L, Werth JL, Poole RM. Clinical importance of changes in chronic pain intensity measured on an 11point numerical pain rating scale. Pain. 2001 ;94(2): 149-158.

https://doi.org/10.1016/s03043959101)00349-9.

33. Vela LI, Denegar CR. The Disablement in the Physically Active Scale, part II: the psychometric properties of an outcomes scale for musculoskeletal injuries. 2010;45(6):63041. https://doi.org/10.4085/1062-605045.6.630.

34. Tanner SM, Dainty KN, Marx RG, J Athl Train Kirkley A. Knee-specific quality-of-life instruments: which ones measure symptoms and disabilities most important to patients? Am J Sports Med. 2007;35(9):1450-1458. doi: $10.1177 / 0363546507301883$.

35. Nirschl RP, Ashman ES. Elbow tendinopathy: tennis elbow. Clin Sports Med. 2003;22(4):813-836. https://doi.org/10.1016/S02785919(03)00051-6.

36. Blazina ME, Kerlan RK, Jobe FW, Carter VS, Carlson GJ. Jumpers Knee. Orthop Clin North Am. 1973;4(3):665-678.

Copyright $($ ) by Indiana State University All rights reserved. ISSN Online 2577-8188
37. Young $M$, Cook J, Purdam C, Alfredson $\mathrm{H}$. Eccentric decline squat protocol offers superior results at 12 months compared with traditional eccentric protocol for patellar tendinopathy in volleyball players. $\mathrm{Br} J$ Sports Med.2005;39:102-105.

https://doi.org/10.1136/bjsm.2003.01058 7.

38. Ohberg L, Alfredson H. Effects on neovascularisation behind the good results with eccentric training in chronic mid-portion Achilles tendinosis? Knee Surg Sports Traumatol Arthrosc. 2004;12(5):465-70. https://doi.org/10.1007/s00167-0040494-8.

39. Cannell LJ, Taunton JE, Clement DB, Smith C, Khan KM. A randomised clinical trial of the efficacy of drop squats or leg extension / leg curl exercises to treat clinically diagnosed jumper's knee in athletes: pilot study. $\mathrm{Br} J$ Sports $\quad$ Med. 2001;35:60-64. https://doi.org/10.1136/bjsm.35.1.60.

40. Jonsson $P$, Alfredson $H$. Superior results with eccentric compared to concentric quadriceps training in patients with jumper's knee: $A$ prospective randomised study. $\mathrm{Br} J$ Sports Med.

2005;39:847-850. https://doi.org/10.1136/bjsm.2005.01863 o.

41. Vicenzino, B., \& Wright, A. Effects of a novel manipulative physiotherapy technique on tennis elbow: a single case study. Man Ther. $1995 ; 1: 30-35$.

https://doi.org/10.1054/math.1995.0247.

42. Amro, A., Diener, I., Bdair, W., Hameda, I., Shalabi, A., \& Ilyyan, D. The effects of Mulligan mobilization with movement and taping technique on pain, grip strength, and function in patients with lateral epicondylitis. Hong Kong Physiotherapy Journal. 2010; 28: 19-23.

https://doi.org/10.1016/i.hkpi.2010.11.00 4 .

43. Bisset L., Beller E., Jull, G., Brooks, P., Darnell, R., \& Vicenzino, B. Mobilisation with movement and exercise, corticosteroid injection, or wait 
and see for tennis elbow: randomised trial.

BJM. 2006;333: 939-941.

https://doi.org/10.1136/bmi.38961.58465

3.ae.

44. Matocha MA, Baker RT, Nasypany AM, Seegmiller JG. Effects of Neuromobilization on Tendinopathy: Part II. Int J Athl Ther Train. $2015 ; 20(2): 41-47$.

https://doi.org/10.1123/isr.2016-0033. 